\title{
"Validación de Ensilaje Elaborado a partir de Pulpa de Café como una Alternativa de Alimentación de Ganado Lechero en Dos Etapas de Experimentación"
}

Blandón, Sandra; Blandón, Marylinda; Torres, Keyla

\section{Resumen}

La investigación contó con el apoyo técnico y financiero de la Fundación para el Desarrollo Tecnológico Agropecuario y Forestal de Nicaragua (FUNICA) y su finalidad fue validar el ensilaje elaborado a partir de pulpa de café en ganado lechero. Dicho estudio se hizo en dos etapas, una de validación en nueve fincas, después de 60 días de ensilaje y la segunda, validación del alimento después de nueve meses de ensilaje en una finca del municipio de Estelí. Para la elaboración se utilizó pulpa fresca, la cual se preparó en dos silos tipo montón. Después de 60 días de ensilaje se realizaron análisis bromatológicos a la pulpa ensilada siendo los contenidos de cafeína de 0.75 y 0.76 . Se inició la validación en fincas, utilizando un diseño "Cross Over", con seis vacas lecheras. Los tratamientos fueron alimentación con pulpa ensilada y manejo tradicional. Se midió el volumen y peso de la leche. De manera global se encontró diferencia entre los tratamientos, a favor de la pulpa de café. En el caso del análisis sensorial, se presentó variación únicamente en el color de la leche proveniente de ganado alimentado de manera tradicional, que resultó más amarillenta. En la segunda etapa, se usaron dos tratamientos, uno fue la inclusión de pulpa de café y el otro fue concentrado comercial (16\% de proteína). Los resultados señalan apropiado el uso de la pulpa de café como alimento para ganado lechero, porque no hubo diferencia entre los tratamientos y por lo tanto, el producto puede competir con el concentrado comercial. Lo mismo ocurrió en el análisis sensorial y en la composición de la leche, ya que no hubo diferencia significativa en las concentraciones de proteínas, grasas y materia seca. Asimismo, se determinó ventaja económica del uso del ensilaje como alimento en comparación al concentrado comercial, con ahorros de C\$8.29 diarios y de C\$1492.2 en seis meses, por cada vaca. Se concluyó que la pulpa de café ensilada tiene efectos positivos sobre la producción de leche y existe disponibilidad de equipos $y$ materiales en el mercado nacional para elaborar el producto.

Palabras clave: pulpa de café, ensilaje, leche, alimento

\section{Introducción}

La presente investigación contó con el apoyo técnico y financiero de FUNICA, en el marco del proyecto "Desarrollo de productos a partir de los desechos del café y validación de pequeñas estructuras de beneficio húmedo", de la alianza UNI FUNICA.

Para el desarrollo del estudio se tomó en cuenta que los residuos sólidos del beneficiado húmedo del café constituyen aproximadamente el $40 \%$ de la cereza y son utilizados en el mundo para la producción de biogás, obtención de abono orgánico, producción de hongos comestibles, obtención de alcohol, vinos, alimentación animal, fabricación de carbón activado, entre otros. 
Para poder aprovechar la pulpa de café en alimentación animal, es necesario emplear métodos de procesamiento que mantengan o mejoren su valor nutritivo sin aumentar excesivamente el costo del producto final. Un proceso que ofrece grandes perspectivas de poder ser aplicado exitosamente en el propósito mencionado es el ensilaje.

Rajkumar Rathinavelu y Giorgio Graziosi, 2005, plantean que la inclusión de pulpa de café ensilada en el régimen alimenticio de algunos animales de granja podría contribuir a reducir los costos de producción de leche y carne, especialmente en los países en desarrollo.

Considerando la información anterior y a partir de la necesidad expresada por los productores de café del Norte de Nicaragua se planteó el presente estudio, cuyo propósito fue validar el ensilaje de pulpa de café como alternativa de alimentación de ganado lechero, para disminuir la contaminación que genera el desecho sólido resultante del beneficiado húmedo.

\section{Materiales y métodos}

La pulpa de café fresca se recolectó de los beneficios "La Trampa" y "San Francisco", de Jinotega. El método de ensilaje fue el de tipo montón, siguiendo las recomendaciones de IICA 2000. A continuación se presentan los pasos:

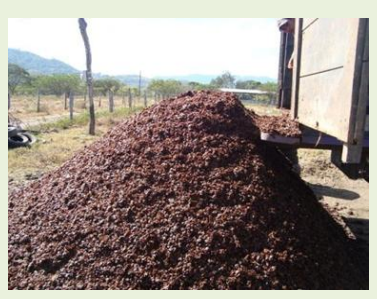

Recepción

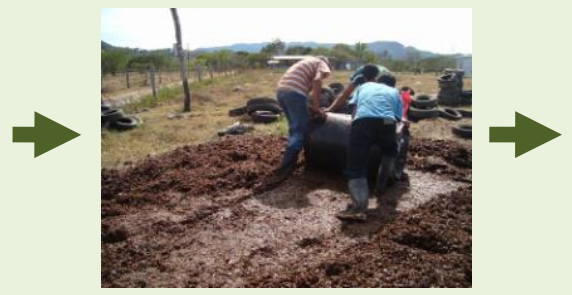

Compactación con barril lleno de agua

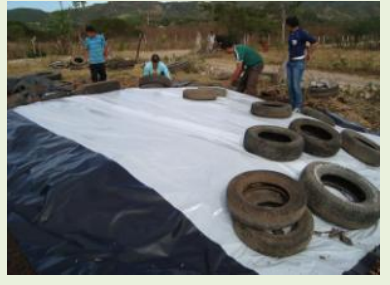

Sellado del silo con llantas usadas y tierra

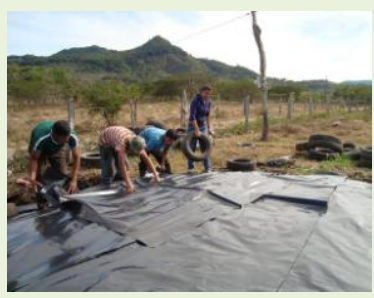

Tapado con plástico-
negro calibre 600

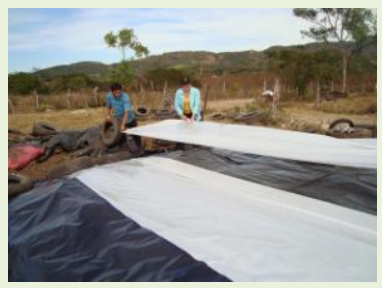

Tapado del silo con plástico blanco

La primera etapa del estudio se llevó a cabo en nueve fincas productoras de leche ubicadas en la parte norte del país, en el departamento de Estelí, Abril a Junio del 2011. Se escogió este ciclo del año por la disponibilidad de la pulpa de café para el ensilaje. 
Los tratamientos de esta etapa fueron alimentación con pulpa ensilada y manejo tradicional y en cada finca se utilizó un diseño "Cross Over", con seis vacas lecheras.

La segunda etapa se llevó a cabo también en época de verano, en el periodo comprendido de octubre a diciembre 2011, en una finca, ubicada en la salida sur de Estelí. Se utilizaron los mismos silos que fueron preparados en la primera etapa y el diseño experimental fue también el mismo, pero los dos tratamientos fueron inclusión de pulpa de café ensilada versus concentrado comercial $(16 \%$ de proteína).

Las variables medidas fueron: volumen, peso de la leche producida. Además del nivel de cafeína en la leche para lo cual se contrató el servicio del Laboratorio de alimentos del Ministerio de Fomento, Industria y Comercio (LABAL MIFIC).

Las propiedades organolépticas de la leche también fueron determinadas, para compararlas entre los tratamientos aplicados. Se tomaron muestras de leche de una de las fincas, medio litro por cada vaca, para un total de 6 muestras que se sometieron a análisis sensorial. El panel estuvo integrado por 10 personas, que tenían como característica principal el ser consumidores de leche. Este análisis de las propiedades organolépticas de la leche se hizo en las dos etapas de la investigación, con la misma metodología.

En cuanto a las propiedades químicas de la leche, se realizaron análisis bromatológicos en el LABAL-MIFIC, para lo cual se tomaron seis muestras de leche procedente de vacas que no consumían pulpa, posteriormente estas vacas se sometieron a la alimentación suplementada con pulpa y se tomaron las muestras para su análisis. De esta manera se determinaron los porcentajes de proteína, grasa, calcio, carbohidratos y materia seca.

Los costos de producción se calcularon tomando en cuenta todos los gastos tanto directos como indirectos que se incurren en la elaboración del silo de pulpa de café; la cantidad de materia prima a utilizar, equipos necesarios, mano de obra requerida, análisis bromatológicos como herramienta en el control de la calidad, depreciación y gastos de operación.

Para estipular el costo unitario por unidad producida se dividió el costo total de producción entre el número de unidades elaboradas; con este valor se comparó el silo con el costo del concentrado comercial.

Los datos que se obtuvieron en el periodo de validación del ensilaje, se analizaron mediante el uso de un Programa estadístico de Ciencias Sociales (SPSS Statistics, 17.0).

\section{Resultados y discusión}

Después de aplicada la metodología en el estudio, se presentan los resultados de cada uno de las etapas de la investigación, las que comprenden el uso de pulpa ensilada después de 60 días y nueve meses, respectivamente. 


\section{Primera etapa}

El promedio de proteína en la pulpa de café ensilada fue de $15 \%$ después de 60 días. Por otra parte, el contenido de cenizas se incrementó después del tiempo de fermentación. Se detectaron diferencias entre los diferentes valores de cenizas siendo $8.93 \%$ en el silo A y $9.14 \%$ en el silo B en la pulpa fresca y $11.79 \%, 11.55 \%$ respectivamente después del ensilado.

Resultados del análisis de los datos recolectados en las nueve fincas

La figura 1 muestra de manera global las diferencias existentes por tratamiento aplicado para la variable peso.

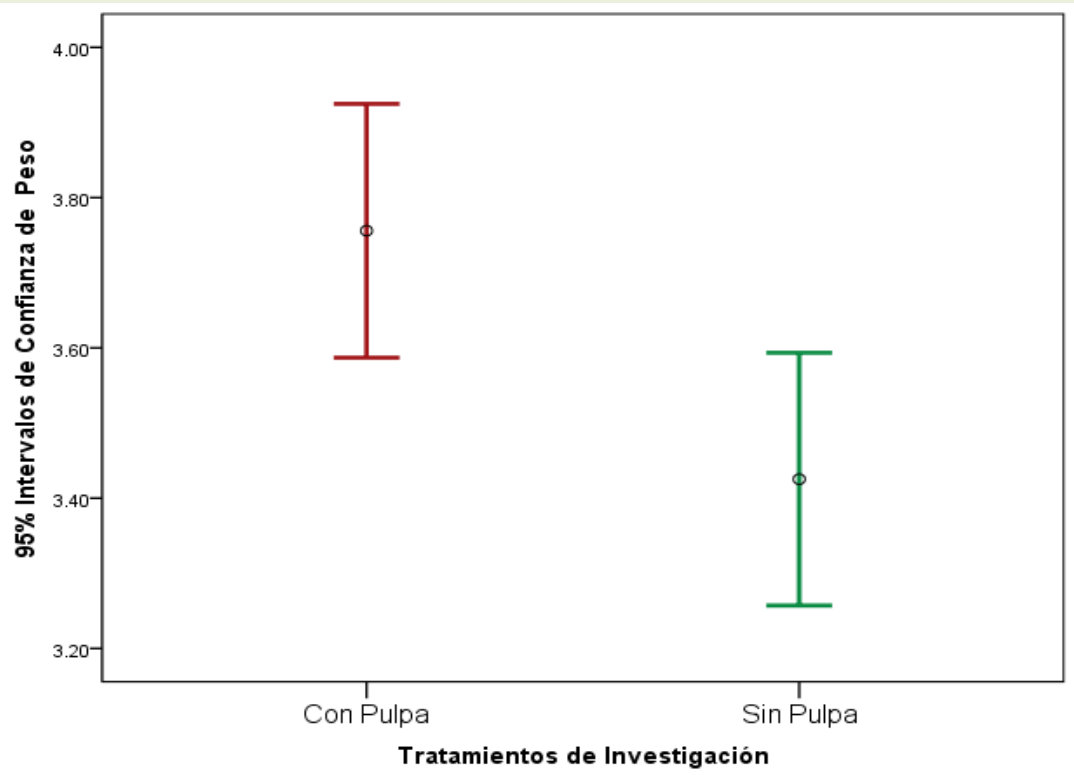

Figura 1. Diferencia de Peso en la leche por tratamiento aplicado

De manera general, para la variable peso se encontró que hay diferencia entre los tratamientos con un grado de significancia de 0.007. Asimismo, los promedios de producción con pulpa son superiores en comparación al tratamiento sin pulpa. La mayoría de los datos para esta variable en las vacas alimentadas sin pulpa oscilaron entre 3.3 y $3.6 \mathrm{~kg}$ y en el tratamiento con pulpa fueron de 3.6 y $4 \mathrm{~kg}$.

En la figura siguiente se muestra la diferencia de volumen en la leche por tratamiento aplicado. 


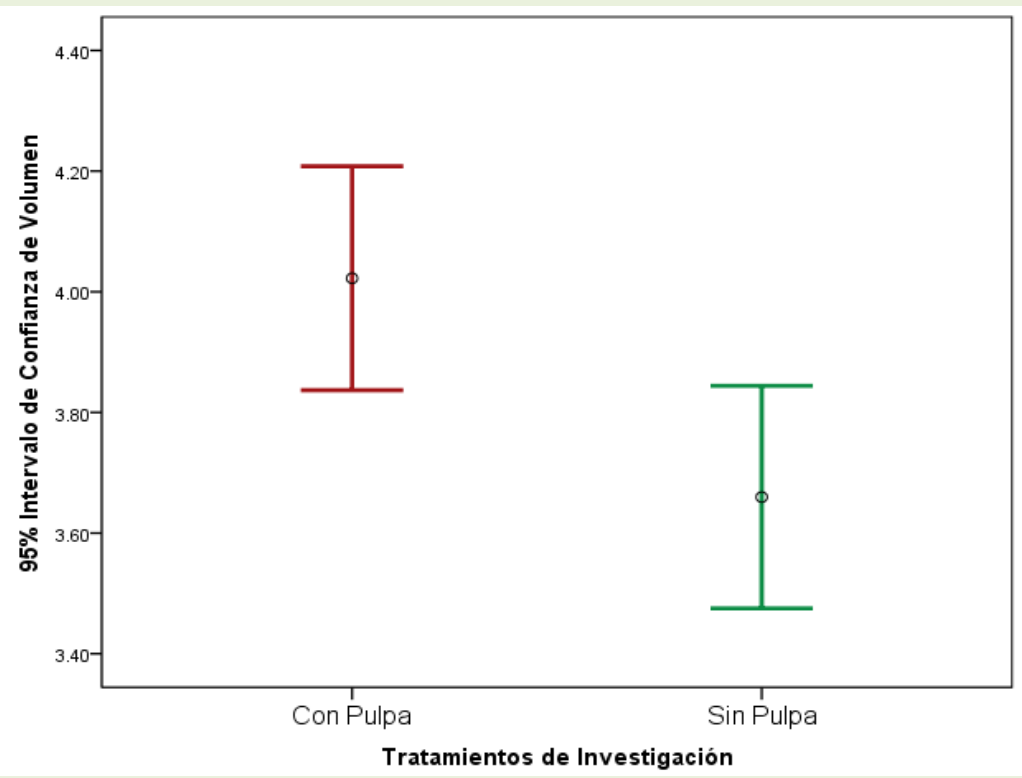

Figura 2. Diferencia de volumen en la leche por tratamiento aplicado

La mayoría de los datos del volumen de la leche de las vacas alimentadas con pulpa oscilaron entre 3.8 y 4.20 litros. Por otra parte, los datos de los animales sometidos a dietas tradicionales que no incluyen ensilaje de pulpa de café, están entre 3.5 y 3.8 litros.

\section{Segunda etapa}

En la figura 3 se pone en evidencia que la prolongación del periodo de ensilaje de 60 días a nueve meses no causa relevancia en los resultados de las medias del volumen para los tratamientos (Con pulpa, Con concentrado).

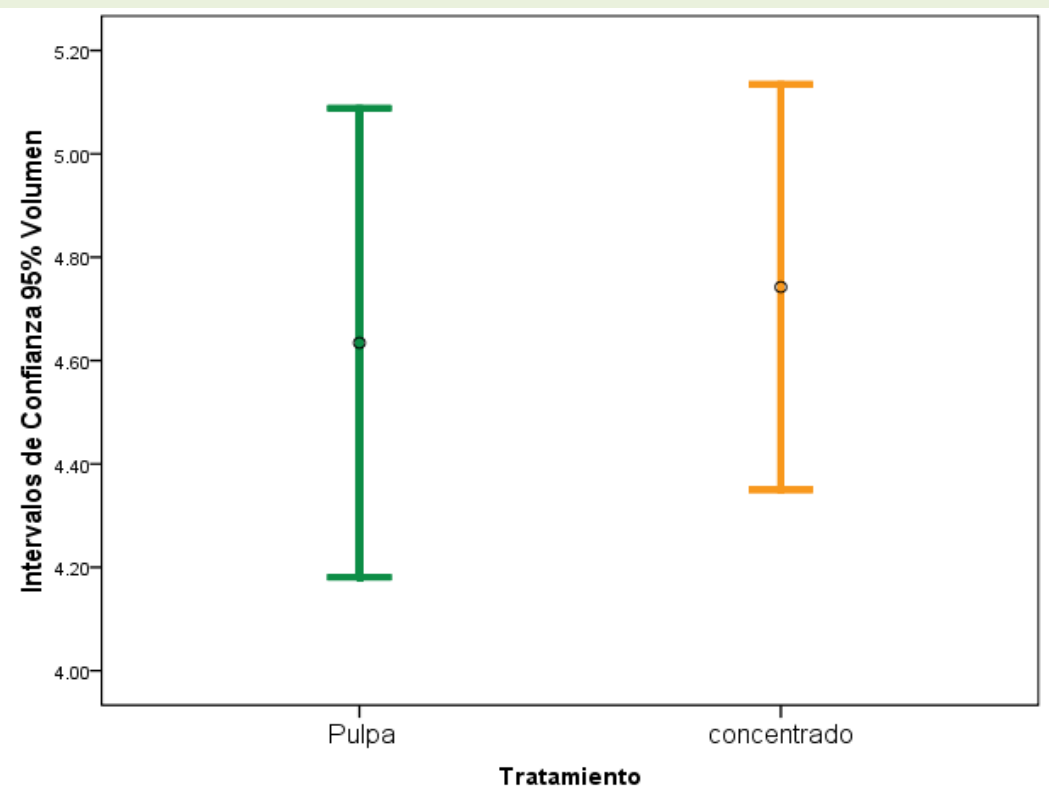

Figura 3. Diterencia de volumen en leche por tratamiento aplicado posterior a nueve meses de ensilaje de pulpa de café en la Finca "Los Jobos" 
Del análisis de varianza univariante se determinó que el nivel de significancia fue mayor a 0.05 , siendo 0.718; encontrándose en hipótesis nula. Por otro lado, el hecho de que las medias para ambos tratamientos sean 4.63 con pulpa y 4.74 con concentrado, no arrojan contraste significativo.

En la figura 4 se detallan los resultados obtenidos del peso de la leche procedente de los bovinos de la finca "Los Jobos".

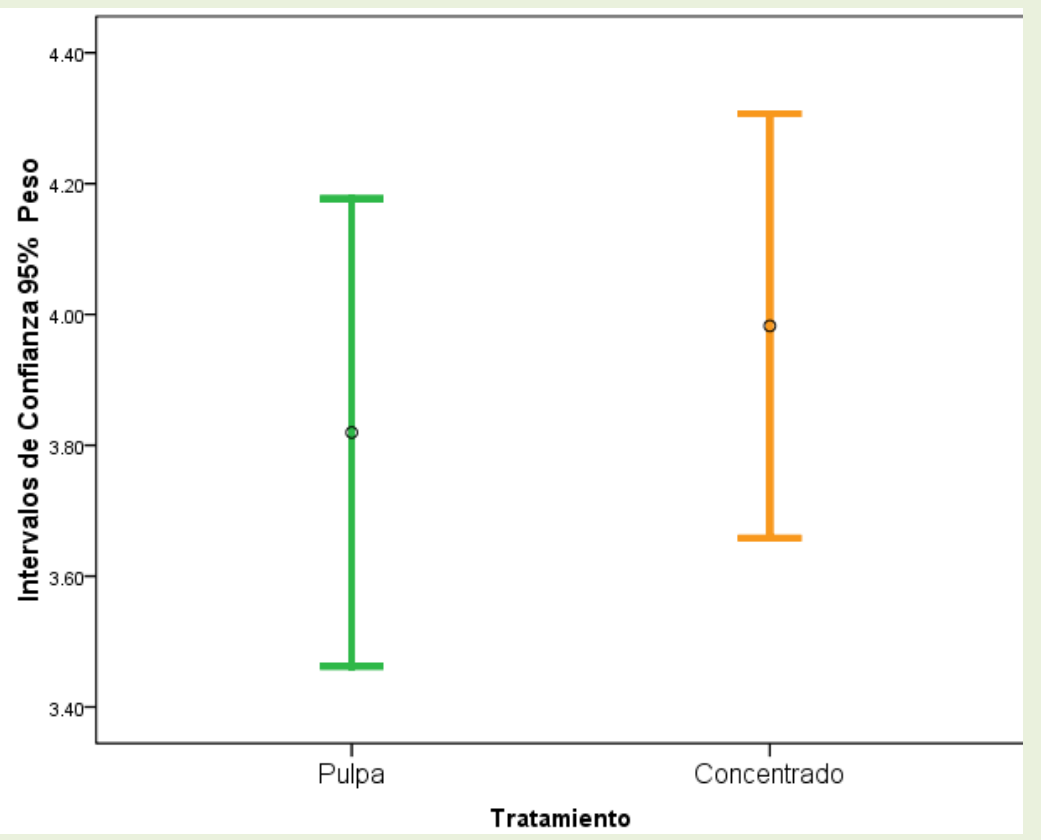

Figura 4. Diferencia de peso en la leche por tratamiento después de transcurrido los nueve meses de ensilaje en la Finca "Los Jobos"

Este caso es similar al anterior; las medias resultantes para peso para el tratamiento con pulpa fue 3.81 , con concentrado 3.98 .

Estos resultados son favorables para recomendar el uso de la pulpa de café como alimento para ganado lechero, ya que se evidencia que este producto puede competir con el concentrado comercial.

\section{Análisis Sensorial}

Al comparar estos resultados con los del primer análisis sensorial (el practicado usando pulpa ensilada de 60 días), se determinó que el color se afecta menos con el incremento del periodo de ensilaje, ya que en el primer análisis se encontró que los valores estaban en los extremos de amarillenta (Tratamiento sin pulpa) y blanca (tratamiento con pulpa). Por otra parte, las propiedades como el sabor y aroma son percibidas normales.

\section{Análisis bromatológico de la leche}

Se determinó el porcentaje de humedad, proteína, grasa, ceniza, carbohidratos, fibra, calcio y materia seca. De la prueba de Chi cuadrado de los resultados se 
encontró que no hay diferencia entre los tratamientos (Con pulpa y Sin pulpa). Además, en la determinación de la cafeína, el resultado fue "ausencia" de este compuesto en las muestras de leche.

\section{Costos de producción}

El costo de producción de una ración de pulpa de café ensilada cuesta $C \$ 11.96$ y el concentrado comercial con $16 \%$ de contenido proteico tiene un costo de C $\$$ 20.25. Si el productor decide alimentar el ganado con pulpa de café ensilada podría ahorrar C $\$ 8.29$ diarios y C $\$ 1492.2$ en seis meses, por cada animal.

\section{Conclusiones}

Finalizado el proceso de validación de la pulpa de café como alimento para ganado lechero se concluye que:

Los tiempos de ensilaje influyen en la composición final del silo, evidenciándose un descenso de la cafeína y aumento de contenido proteico.

El ensilaje de pulpa de café resulta una alternativa viable de alimentación para ganado lechero, ya que comparado con la dieta tradicional, hay diferencias significativas entre los tratamientos, a favor de la pulpa.

Después de nueve meses de ensilaje y comparando dos tratamientos, la pulpa de café y el concentrado comercial, se demostró que no hay diferencia significativa entre los tratamientos. Por lo que la pulpa de café puede sustituir el concentrado comercial ( $16 \%$ de proteínas) en la dieta del ganado lechero.

La dieta con pulpa de café ensilada no ejerce efectos sobre las propiedades químicas y sensoriales de la leche. Las pruebas de significancia fueron mayores a 0.05 .

Existe ventaja económica en el uso de la pulpa de café como alimento para ganado lechero. La ración de pulpa de café ensilada cuesta C\$11.96 y el concentrado comercial (16\% de proteína) tiene un costo de C\$20.25. Además, existe disponibilidad de equipos y materiales en el mercado nacional para elaborar el producto.

\section{Referencias}

IICA. (2000). El ensilaje en la alimentación de ganado vacuno. En G. Chaverra, \& J. Bernal. Colombia: Tercer Mundo Editores.

Rajkumar Rathinavelu y Giorgio Graziosi. (17 de Agosto de 2005). Posibles usos alternativos de los residuos. Recuperado el 25 de septiembre de 2010, de Posibles usos alternativos de los residuos: http://www.ico.org/documents/ed1967c.pdf 